ARTICLE

DOI: $10.1038 / \mathrm{s} 41467-018-05010-0$

\title{
XPC is an RNA polymerase II cofactor recruiting ATAC to promoters by interacting with E2F1
}

\author{
B. Bidon (10 1,2,3,4, I. Iltis ${ }^{1,2,3,4}$, M. Semer ${ }^{1,2,3,4}$, Z. Nagy ${ }^{1,2,3,4}$, A. Larnicol ${ }^{1,2,3,4}$, A. Cribier ${ }^{5}$, M. Benkirane ${ }^{5}$, \\ F. Coin ${ }^{1,2,3,4}$, J-M. Egly ${ }^{1,2,3,4}$ \& N. Le May (1) 1,2,3,4
}

The DNA damage sensor XPC is involved in nucleotide excision repair. Here we show that in the absence of damage, XPC co-localizes with RNA polymerase II (Pol II) and active posttranslational histone modifications marks on a subset of class II promoters in human fibroblasts. XPC depletion triggers specific gene down-expression due to a drop in the deposition of histone $\mathrm{H} 3 \mathrm{~K} 9$ acetylation mark and pre-initiation complex formation. XPC interacts with the histone acetyltransferase KAT2A and specifically triggers the recruitment of the KAT2Acontaining ATAC complex to the promoters of down-expressed genes. We show that a strong E2F1 signature characterizes the XPC/KAT2A-bound promoters and that XPC interacts with E2F1 and promotes its binding to its DNA element. Our data reveal that the DNA repair factor XPC is also an RNA polymerase II cofactor recruiting the ATAC coactivator complex to promoters by interacting with the DNA binding transcription factor E2F1.

\footnotetext{
${ }^{1}$ Institut de Génétique et de Biologie Moléculaire et Cellulaire, BP 163 IIlkirch Cedex, 67404 C.U. Strasbourg, France. ${ }^{2}$ Centre National de la Recherche Scientifique, UMR7104, 67404 IIlkirch, France. ${ }^{3}$ Institut National de la Santé et de la Recherche Médicale, U1258, 67404 IIlkirch, France. ${ }^{4}$ Université de Strasbourg, 67404 IIlkirch, France. ${ }^{5}$ Institut de Génétique Humaine, Laboratoire de Virologie Moléculaire, Université de Montpellier, CNRS UMR9002, 34000 Montpellier, France. These authors contributed equally: B. Bidon, I. Iltis. Correspondence and requests for materials should be addressed to F.C. (email: fredr@igbmc.fr) or to J.-M.E. (email: egly@igbmc.fr) or to N.L.M. (email: nlemay@igbmc.fr)
} 
G ene expression is constantly compromised by genotoxic stress that challenges genome integrity and requires the function of several DNA repair pathways to remove DNA lesions. This implies that there must be connections between the disparate events of transcription and DNA repair to orchestrate the expression and repair of genes. A link between DNA repair and transcription was first established after the discovery of a nucleotide excision repair (NER) sub-pathway removing DNA lesions located on the actively transcribed strand blocking elongating RNA polymerase II (Pol II) called the transcription coupled repair (TCR $)^{1}$. This was followed by the characterization of the basal transcription TFIIH as a NER factor involved both in TCR and in global genome repair (GGR), eliminating DNA damage from the entire genome $\mathrm{e}^{2,3}$. This interplay is even tighter, since studies also revealed roles for the other NER factors (CSB, XPC, XPA, XPG, and XPF/ERCC1) in gene expression ${ }^{4-6}$.

Understanding the roles played by NER factors is of prime importance not only to unveil the molecular details of gene expression but also to understand how mutations in their corresponding genes give rise to several human autosomal recessive disorders like Xeroderma pigmentosum (XP), Cockayne syndrome (CS), and trichothiodystrophy (TTD). Patients bearing mutations in XPC only develop XP (XP-C) and represent the most frequent NER-defective group. XP is clinically characterized by an extreme sensitivity to ultraviolet (UV) rays from sunlight. XP patients develop severe sunburns and are highly susceptible to develop tumors on sunlight-exposed areas of the skin, including melanoma and squamous cell carcinoma ${ }^{7}$. XP individuals also present increased susceptibility for lung, breast, and colorectal cancers with possible neurological issues ${ }^{8}$. The XP pathology has been primarily defined as a DNA repair syndrome due to the inability of patients' cells to eliminate DNA lesions. However, studies during the last decade suggest that some of their phenotypes may also stem from transcriptional deregulations ${ }^{9}$.

Upon NER, XPC, with its partner hHR23B, recognizes all along the genome DNA-distorting lesions inflicted by endogenous and exogenous genotoxic attacks like UV irradiation, thereby initiating only the GGR sub-pathway ${ }^{10}$. Several observations suggest that XPC is additionally involved in the modifications of the chromatin environment surrounding the DNA lesions, including histone post-translational modifications $(\mathrm{PTMs})^{11-13}$. We have shown earlier that NER factors are associated with the Pol II transcription machinery and are sequentially recruited at the promoter of transcribed genes ${ }^{6}$. The presence of these NER factors at promoter is required to achieve optimal chromatin remodeling, including histone PTMs as well as active DNA demethylation, DNA break induction, and gene looping6,14. Furthermore, a complex containing XPC and Oct4/Sox 2 has been identified as a coactivator in embryonic stem (ES) and induced pluripotent stem cells ${ }^{15,16}$. Although the involvement of XPC in transcription is established, its mechanistic role remains largely elusive as well as its transcriptional partners in the pre-initiation complex.

In the present study, we investigated the roles of XPC in class II gene expression. We first assessed the genome-wide localization of XPC and revealed that in the absence of a genomic stress, XPC is mainly recruited to the promoters of active genes where it colocalizes with Pol II. Depletion of XPC leads to deregulation of Pol II recruitment and altered histone marks at promoters, including $\mathrm{H} 3 \mathrm{~K} 9 \mathrm{ac}$. We further identified an interaction between $\mathrm{XPC}$ and the histone acetyltransferase (HAT) lysine acetyltransferase 2A KAT2A (or GCN5). Our data indicated that XPC, through its interaction with KAT2A, could be associated with both ATAC and SAGA complexes but that only ATAC is detected at the promoters of XPC-dependent genes. GREAT analysis unveiled that a strong E2F1 signature characterizes genes regulated by XPC/KAT2A. We showed that E2F1 interacts with $\mathrm{XPC}$ and is required for its localization at the promoter of activated genes. We subsequently identified a complex, including $\mathrm{XPC}, \mathrm{E} 2 \mathrm{~F} 1$, and KAT2A that is specifically recruited to XPCdependent promoters, conditioning the presence of the ATAC complex and the deposition of the $\mathrm{H} 3 \mathrm{~K} 9 \mathrm{ac}$ mark. Altogether, our results establish XPC as a cofactor of Pol II, interacting with a transcription factor and recruiting a coactivator complex to remodel chromatin and initiate class II gene expression.

\section{Results}

Enrichment of XPC on promoters in the absence of genomic stress. To investigate the involvement of XPC upon transcription, we set forward to analyze transcription genome-wide in fibroblasts defective in endogenous XPC, hereafter called XP-C ${ }^{\mathrm{DEL}}$ cell line (Fig. 1a) ${ }^{17,18}$. Wild-type GFP-XPC cDNA was re-introduced into XP-C $\mathrm{C}^{\mathrm{DEL}}$ cells to be used as positive control cell line, hereafter called XP-C ${ }^{\mathrm{WT} 19}$. All along the study, we also compared these two cell lines to another XP-C patient cell line bearing the XPC p.Pro334His mutation with mild phenotypes, in which XPC is mutated and expressed (Fig. 1a) and hereafter called XP$\mathrm{C}^{\text {MUT17 }}$

By employing the all-trans retinoic acid (ATRA) stimulus, which induces a well-characterized modification of the cellular transcriptional program ${ }^{6,14}$, we sought to identify the XPC genomic distribution and its impact on gene expression in a welldefined system. We first analyzed the RA response in the three cell lines by studying the transactivation of RARß2 upon ATRA treatment. We observed a correlation at $6 \mathrm{~h}$ post ATRA treatment between $R A R \beta 2$ mRNA induction and recruitment of the transcriptional machinery (including RAR, Pol II, and TFIIH kinase CDK7), together with the NER factors (XPC, XPA, and $\mathrm{XPG}$ ) at the RARß2 promoter in both $\mathrm{XP}-\mathrm{C}^{\mathrm{WT}}$ and $\mathrm{XP}-\mathrm{C}^{\mathrm{MUT}}$ cells (Fig. $1 \mathrm{~b}$ and Supplementary Figure 1A). However, RARß2 transactivation and the concomitant recruitment pattern were largely abolished in XP-C $\mathrm{C}^{\mathrm{DEL}}$ cells characterized by the absence of XPC (Fig. 1b and Supplementary Figure 1A). We thus performed chromatin immunoprecipitation (ChIP) followed by highthroughput DNA sequencing (ChIP-seq) in both XP-C ${ }^{\mathrm{WT}}$ and $\mathrm{XPC} \mathrm{DEL}^{\mathrm{CEl}}$ cells that were treated during $6 \mathrm{~h}$ with ATRA using antibodies directed against green fluorescent protein (GFP) and Pol II. Three independent series using XP-C ${ }^{\mathrm{WT}}$ cells yielded 2191 and 16, 440 peaks for GFP-tagged XPC and Pol II, respectively. We identified 1797 common binding events representing 82\% of the XPC peaks (Fig. 1c). Using HOMER annotation, we observed that $32 \%$ of the XPC-binding events appeared at promoter regions (Fig. 1d). Moreover, the combination of $5^{\prime}$ untranslated region and exon-annotated peaks (mostly located in the very first one) represented $44 \%$ of the XPC peaks, all located near the transcription starting site (TSS). Interestingly, $94 \%$ of these XPC-bound promoters also harbored an enrichment of Pol II (Fig. 1d).

RNA-seq analysis has been performed in parallel to ChIP-seq in the ATRA-treated XP-C ${ }^{\mathrm{DEL}}$ cells and compared to XP-C $\mathrm{C}^{\mathrm{WT}}$. Peak annotation of the 1797 common binding events between XPC and Pol II identified 1529 genes, whose expression varied after ATRA treatment indicating that they were presumably regulated by the presence of XPC. We observed that 283 genes were downregulated in the absence of XPC, whereas 241 genes were upregulated (Fig. 1e and Supplementary Data 1). Based on these results, we defined two groups of genes: one containing 283 genes positively regulated by XPC and the other containing 241 genes negatively regulated by XPC. The top-ranking Gene Ontology (GO) biological process with significant $p$ values included chromatinstructure-related terms such as chromatin/nucleosome assembly, 
DNA packaging, and protein-DNA organization that is in agreement with the well-defined role of XPC in genomic stability (Supplementary Figure 1B). As expected, the top-ranking GO biological pathways were related to RAR-targeted genes but we also noticed numerous interferon (IFN) gamma-related genes (Supplementary Data 2).
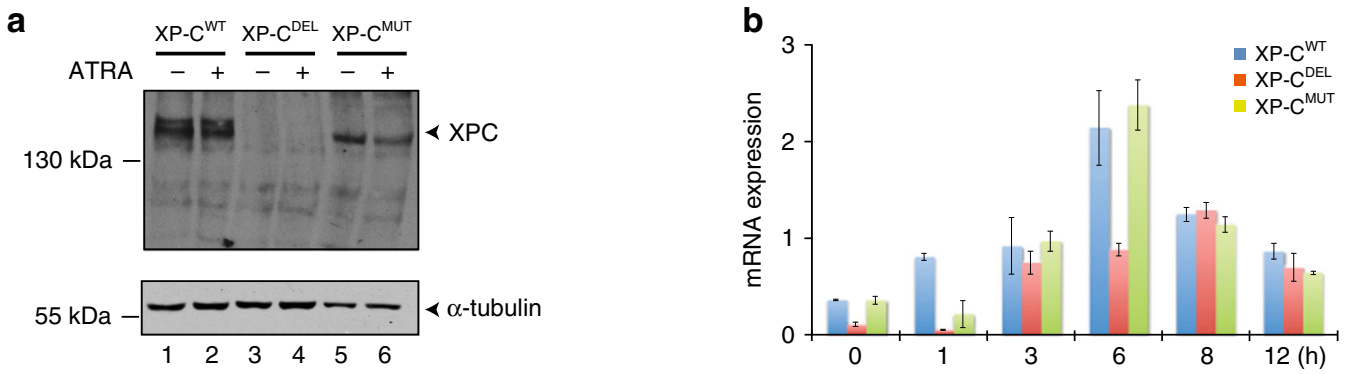

C

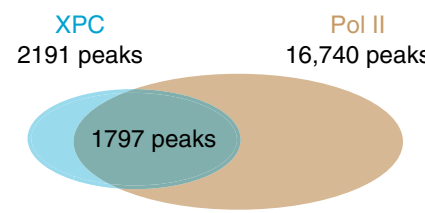

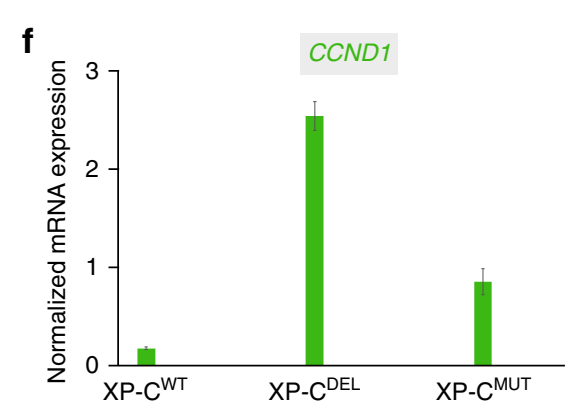

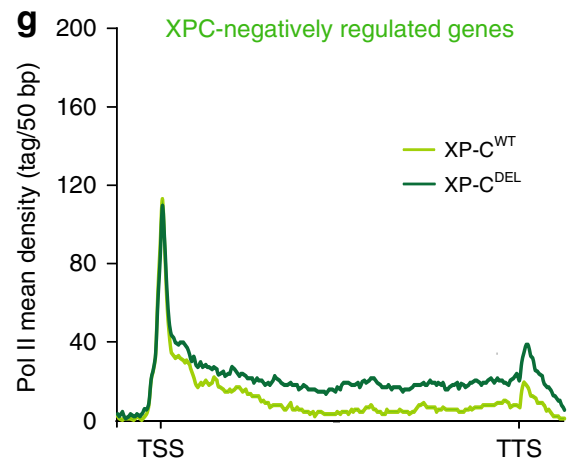

d
Intergenic

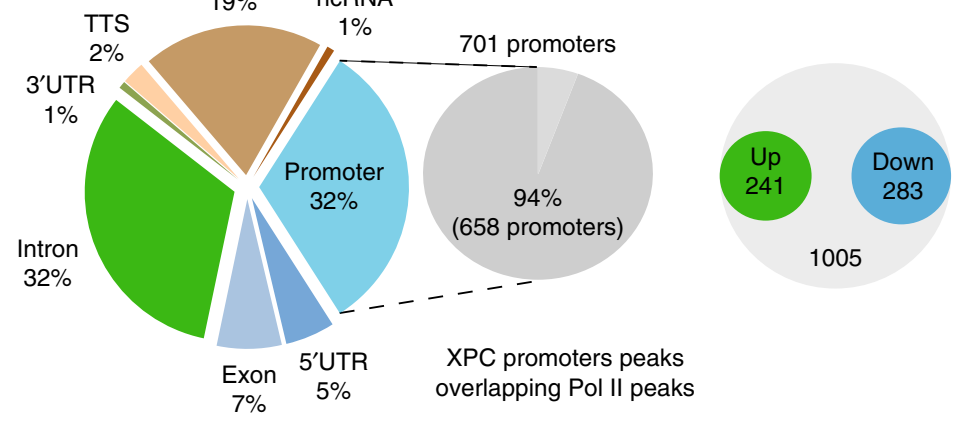

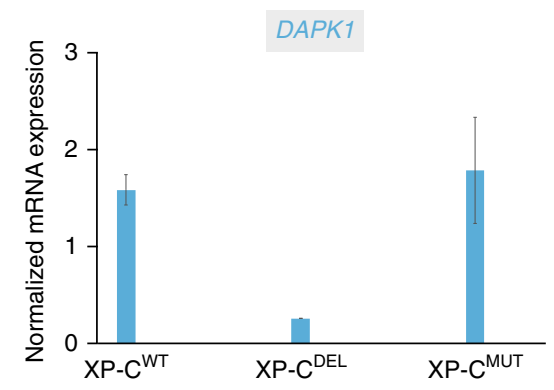

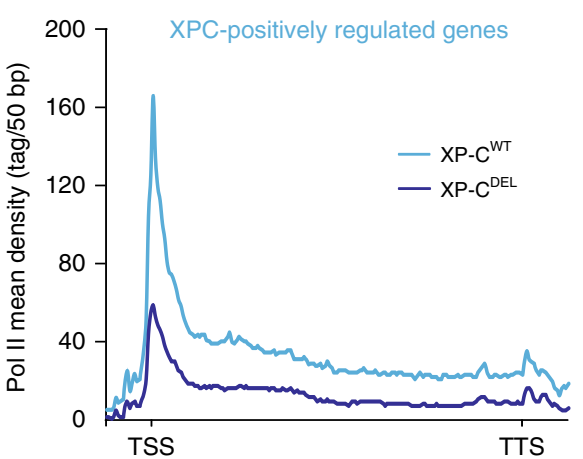

Fig. 1 Co-localization of XPC and Pol Il genome-wide. a Relative protein expression of XPC and $\alpha$-tubulin analyzed by western blotting of whole-cell extract from XP-CWT, XP-C $C^{D E L}$, and XP-CMUT fibroblasts, in presence or absence of ATRA. b Relative mRNA expression of RARB2 in XP-CWT, XP-C $C^{D E L}$, and XPCMUT fibroblasts, after ATRA treatment in 12-h time-course experiment. Error bars represent the standard deviation of three independent experiments. $\mathbf{c}$ Overlapping of MACS14-determined peaks for both Pol II and XPC in XP-CWT cells after ATRA induction. XPC and Pol II peaks correspond to recurrent peaks found in three independent ChIP-seq experiments. d HOMER annotation of the 2191 XPC peaks and proportion of promoter annotated peaks to overlap with a Pol II peak. e Expression level of XPC-bound genes identified by ChIP-seq were determined by RNA-seq by comparing both ATRA-treated $X P-C^{W T}$ and $X P-C^{D E L}$ cells. Differential expression analysis of these genes in XP-CWT, compared to XP-CDEL, was then computed using EdgeR represented as a circle plot. XPC-negatively regulated genes (upregulated in XP-C $C^{D E L}$ ) are enlightened in green and XPC-positively regulated genes (downregulated in $\left.X P-C^{D E L}\right)$ in blue. $f$ Relative mRNA expression of two genes, CCND1 as XPC-negatively regulated one and DAPK1 as XPC-positively regulated one, after ATRA treatment in XP-CWT, XP-CDEL, and XP-CMUT cells. Error bars represent the standard deviation of three independent experiments. $\mathbf{g}$ Diagrams representing the mean tag density of Pol II ChIP-seq experiment for XPC-negatively regulated and -positively regulated genes, as they were previously determined in XP-CWT and XP-CDEL cells 
We subsequently validated RNA-seq by reverse transcription-PCR in XP-C $\mathrm{C}^{\mathrm{WT}}$ and $\mathrm{XP}-\mathrm{C}^{\mathrm{DEL}}$ cells, using $C C N D 1$ as representative of $\mathrm{XPC}$-negatively regulated genes and DAPK1, HOXB13, and $L R R C 11$ as representatives of XPCpositively regulated genes and observed that the expression of these genes was not modified by the XPC p.Pro334His mutation found in XP-C ${ }^{M U T}$ cells (Fig. If and Supplementary Figure 1E).

We next determined the mean tag density of Pol II for both $\mathrm{XPC}$-positively and -negatively regulated genes in XP-C ${ }^{\mathrm{DEL}}$ cells. We observed a lower occupancy of Pol II at TSS and along the gene body of the XPC-positively regulated genes in the absence of XPC (Fig. 1g, right panel), while Pol II enrichment was higher mainly along the gene body for the XPC-negatively regulated genes (Fig. 1g, left panel). ChIP experiments targeting the CCND1 or DAPK1 promoter recapitulated ChIP-seq data (see also additional XPC-positively regulated genes in Supplementary Figure $1 \mathrm{~F}$, top panels). Finally, to rule out the possibility that our observations were due to an overexpression of XPC in the rescued XP-C $\mathrm{C}^{\mathrm{WT}}$ cells, we tested and compared rescued $\mathrm{XP}-\mathrm{C}^{\mathrm{WT}}$ fibroblasts with MRC- 5 cells showing similar inductions of XPCtarget genes and comparable recruitments by ChIP upon ATRA treatment in these two cell lines (Supplementary Figure 1C-D).

Collectively, our findings established an enrichment of XPC around promoters that strongly correlates with Pol II at TSSs, regulating more than 500 genes.

Enrichment of XPC correlates with positive histone PTMs. We next investigated the correlation between the presence of XPC and the deposition of positive histone marks at TSS. We performed a comparative ChIP-seq analysis using both ATRAtreated XP-C $\mathrm{CHL}^{\mathrm{DE}}$ and $\mathrm{XP}-\mathrm{C}^{\mathrm{WT}}$ cells for $\mathrm{H} 3 \mathrm{~K} 9 \mathrm{ac}$ and $\mathrm{H} 3 \mathrm{~K} 4 \mathrm{me} 3$ marks. Among the 1797 common binding events between XPC and Pol II detected in XP-C ${ }^{\mathrm{WT}}$ cells, 1756 (98\%) were localizing with H3K9ac and 1385 (78\%) with both H3K9ac and H3K4me3 (Fig. 2a). Interestingly, ChIP-seq data comparison between XP$\mathrm{C}^{\mathrm{WT}}$ and XP-C $\mathrm{C}^{\mathrm{DEL}}$ cells, considering previously identified XPCregulated genes, showed a correlation between their expression levels, the enrichment of Pol II, and the deposition of the transcription-positive histone marks. Indeed, the fragment depth for $\mathrm{H} 3 \mathrm{~K} 9 \mathrm{ac}$ and $\mathrm{H} 3 \mathrm{~K} 4 \mathrm{me} 3$ was higher at promoters of the XPCnegatively regulated genes while they remained lower for XPCpositively regulated genes in $\mathrm{XP}-\mathrm{C}^{\mathrm{DEL}}$ cells compared to XP$\mathrm{C}^{\mathrm{WT}}$ cells (Fig. 2b). Distribution of these histones marks along the representative genes CCND1 and DAPK1 is shown in Fig. 2c and ChIP on their promoters in Fig. 2d (see also supplemental genes in Supplementary Figure 1F, lower panels). In contrast, and in agreement with the normal expression of CCND1 and $D A P K 1$ in XP-C ${ }^{\mathrm{MUT}}$ cells compared to XP-C ${ }^{\mathrm{WT}}$ cells (Fig. 1f), the deposition of $\mathrm{H} 3 \mathrm{~K} 9 \mathrm{ac}$ and $\mathrm{H} 3 \mathrm{~K} 4 \mathrm{me} 3$ on the promoter of these genes was not modified in XP-C ${ }^{\text {MUT }}$ cells (Fig. $2 \mathrm{~d}$ and Supplementary Figure 1F, lower panels).

To exclude the false positive results due to the use of $\mathrm{XPC}^{\mathrm{WT}}$ cells overexpressing XPC, we also analyzed HeLa cells constitutively expressing short hairpin RNA either directed against XPC (shXPC), XPA (shXPA), or scrambled (shCtrl) overtime treated with ATRA (Supplementary Figure 2A, left panel). We observed lower induction of mRNA RARß2 synthesis, $6 \mathrm{~h}$ post ATRA treatment in shXPC and shXPA cells, compared with shCTL cells (Supplementary Figure 2A, middle panel). We also determined the transcriptome profiles of shCtrl, shXPC, and XP- $\mathrm{C}^{\mathrm{DEL}}$ cells by comparing mRNA induction in untreated and $6 \mathrm{~h}$ ATRA-treated cells. In shCtrl cells, 77 genes show a fold induction $>2$, including several well-characterized genes, such as $R A R \beta 2$ (Supplementary Figure 2B, Supplementary Data 4, and Supplementary Methods). Surprisingly, $\sim 90 \%$ of genes activated in shCtrl showed a lower
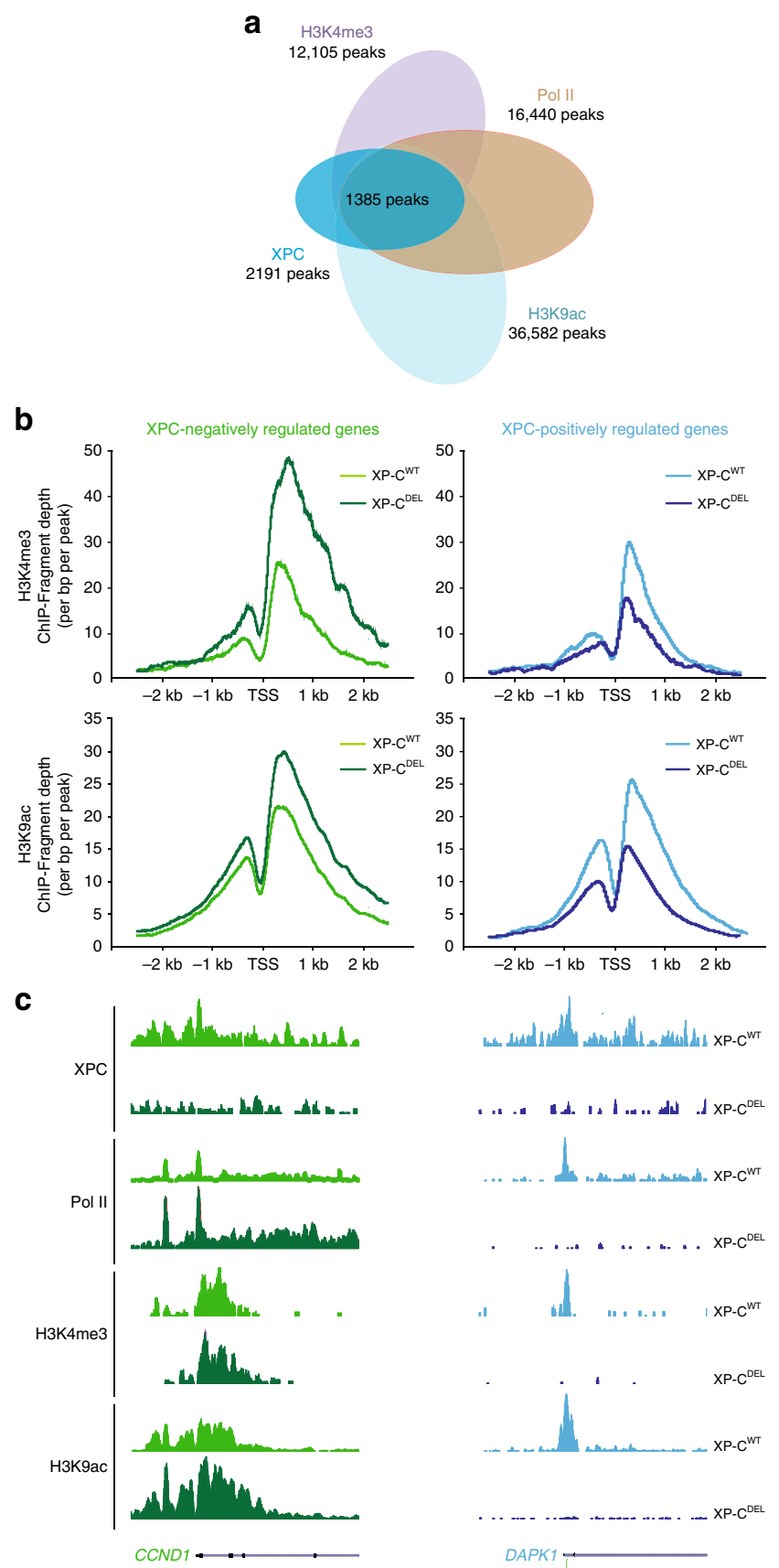

d
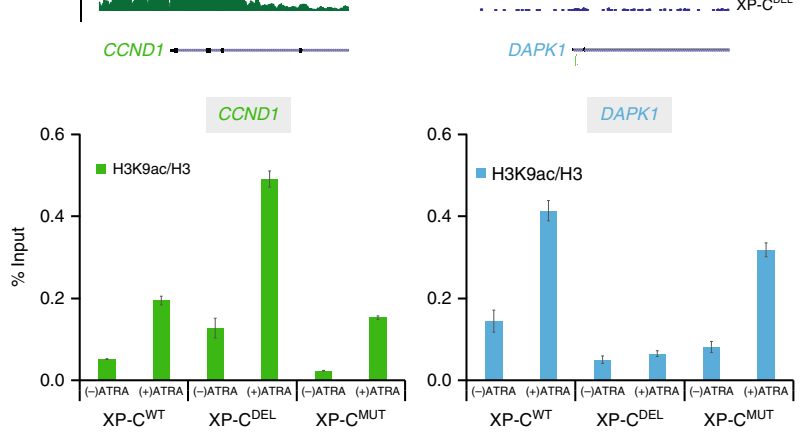

induction in both shXPC and XP-C ${ }^{\mathrm{DEL}}$ cells (Supplementary Figure $2 \mathrm{~B}$ and Supplementary Data 4). The RARß2 mRNA induction at $6 \mathrm{~h}$ post ATRA treatment in shCtrl cells followed the recruitment of the transcriptional machinery together with the NER factors at the promoter (Supplementary Figure 2A, right panels $)^{6}$. This concomitant recruitment pattern was abolished in 
Fig. 2 Association of XPC-bound promoters with active histone PTMs. a Overlapping of peaks for Pol II and XPC with PTMs H3K4me3 and H3K9ac in XP-CWT cells after ATRA induction. These peaks correspond to recurrent peaks found in three independent ChIP-seq experiments. $\mathbf{b}$ Diagrams representing the fragment depth of H3K9ac and H3K4me3 ChIPseq experiment for XPC-negatively regulated (left panels) and XPCpositively regulated (right panels) genes, as they were previously determined in XP-CWT and XP-C $C^{D E L}$ cells. c UCSC genome browser for XPC, Pol II, H3K4me3, and H3K9ac at CCND1 and DAPK1 promoters as $X P C$-negatively regulated and $X P C$-positively regulated genes, respectively. d Relative enrichment of $\mathrm{H} 3 \mathrm{~K} 9 \mathrm{ac} / \mathrm{H} 3$ monitored by $\mathrm{ChIP}$ at CCND1 and DAPK1 promoters as XPC-negatively regulated and XPC-positively regulated genes. Error bars represent the standard deviation of three independent experiments

shXPC and shXPA cells, although XPC was still detected in the latter one (Supplementary Figure 2A, right panels). While the deposition of $\mathrm{H} 3 \mathrm{~K} 9 \mathrm{ac}$ and $\mathrm{H} 3 \mathrm{~K} 4 \mathrm{me} 3$ was detected after ATRA treatment at the promoter upon $R A R \beta 2$ transactivation in shCtrl cells, such enrichment was not observed in shXPC cells. Similar results concerning the deposition of histone marks at $R A R \beta 2$ promoter were obtained in the corresponding $\mathrm{XP}-\mathrm{C}^{\mathrm{WT}}$ and XP$\mathrm{C}^{\mathrm{DEL}}$ cells (Supplementary Figure 2D, right panel). Interestingly, the deposition of the two histone marks was not disturbed by the absence of XPA in shXPA cells in which normal recruitment of XPC was detected (Supplementary Figure 2D, left panel).

Altogether, these results indicated that the genome-wide recruitment of XPC at promoters correlated with transcriptionally active $\mathrm{H} 3 \mathrm{~K} 9 \mathrm{ac}$ and $\mathrm{H} 3 \mathrm{~K} 4 \mathrm{me} 3$ marks. They also indicated that $\mathrm{XPC}$ was recruited to promoters before XPA in order to initiate histone PTMs at the TSS.

XPC and KAT2A are recruited to XPC-positively regulated genes. We next wondered which enzyme(s) could be responsible for the histone marks deposition at the TSS of XPC-regulated genes. Given the role of KAT2A in the deposition of H3K9ac in transcription initiation ${ }^{20}$, we assessed whether the variations of $\mathrm{H} 3 \mathrm{~K} 9 \mathrm{ac}$ at promoters targeted by XPC could be related to this HAT.

We thus performed ChIP-seq in both $\mathrm{XP}-\mathrm{C}^{\mathrm{WT}}$ and $\mathrm{XP}-\mathrm{C}^{\mathrm{DEL}}$ cells treated $6 \mathrm{~h}$ with ATRA, using antibodies directed against KAT2A, and obtained 19,758 genomic locations for KAT2A compared with input. Using HOMER annotation, we observed almost $50 \%$ of these KAT2A-binding events appearing at promoter regions (Supplementary Figure 3A). Among the previously determined XPC and Pol II co-occupied positions, we identified 948 common binding events with KAT2A (Fig. 3a). Interestingly, KAT2A was absent from promoters of the XPCpositively regulated genes in XP- $\mathrm{C}^{\mathrm{DEL}}$ cells lacking XPC, which correlates with a decrease in $\mathrm{H} 3 \mathrm{~K} 9 \mathrm{ac}$ (Fig. 3b, right panel compared with Fig. 2b). Surprisingly, KAT2A was detected around TSS of XPC-positively regulated genes only in the presence of XPC in XP-C ${ }^{\mathrm{WT}}$ cells while the HAT was hardly detected at XPC-negatively regulated genes with or without XPC, in XP-C $\mathrm{C}^{\mathrm{WT}}$, or XP-C $\mathrm{C}^{\mathrm{DEL}}$, respectively (Fig. $3 \mathrm{~b}$ ). Distribution of KAT2A along the representative genes CCND1 and DAPK1 is shown in Fig. 3c. We confirmed by ChIP that KAT2A recruitment correlated with an increase $\mathrm{H} 3 \mathrm{~K} 9 \mathrm{ac}$ at the promoter of DAPK1 in XP-C ${ }^{\mathrm{WT}}$ cells upon ATRA treatment while the HAT was not detected at CCND1 (Fig. 3d compared with Fig. 2d and also Supplementary Figure $3 \mathrm{~B}$ for two additional genes). This recruitment was lost for DAPK1 in the absence of XPC in XP$\mathrm{C}^{\mathrm{DEL}}$ cells concomitantly with the decreased histone PTMs, while we did not observe any change in $\mathrm{XP}-\mathrm{C}^{\mathrm{MUT}}$ cells (Fig. 3d compared with 2D). Interestingly, we did not detect the closely related paralog of KAT2A, KAT2B (or PCAF) ${ }^{20,21}$, on the representative CCND1 and DAPK1 promoters by ChIP (Fig. 3d and Supplementary Figure $3 \mathrm{~B}$ for additional genes). We also detected KAT2A concomitantly with an increase of $\mathrm{H} 3 \mathrm{~K} 9 \mathrm{ac}$ signal at the activated $R A R \beta 2$ promoter in shCTL, XP-C ${ }^{\mathrm{MUT}}$, XP$\mathrm{CWT}^{\mathrm{WT}}$, and shXPA cells (Supplementary Figure 2D). Interestingly, KAT2A was not detected in the absence of XPC in XP-C ${ }^{D E L}$ and shXPC cells (Supplementary Figure 2D). Finally, ChIP-reChIP assays showed that XPC and KAT2A co-occupied RARß2 promoter in shCtrl but not in shXPC cells, using both combinations of co-immunoprecipitation (co-IP; XPC/KAT2A or KAT2A/XPC) (Supplementary Figure 3C). These results are comparable to the XPC/Pol II co-occupancy detected at this promoter (Supplementary Figure $3 \mathrm{C})^{6}$. Note that KAT2A was stable in all the cell lines (Supplementary Figure 2C). Taken together, our results point to a correlation between the recruitment of XPC and KAT2A and the deposition of H3K9ac at promoters of XPC-positively regulated genes.

XPC recruits ATAC to XPC-positively regulated genes. To deepen the correlation between XPC and KAT2A established above, we next assessed their co-location in a complex and their putative interaction. KAT2A co-precipitated a complex containing XPC in nuclear extracts from untreated XP-C $\mathrm{CT}^{\mathrm{WT}}$ (Fig. 4a). As expected, such co-precipitation was not detected using nuclear extracts from untreated XP-C ${ }^{D E L}$. Furthermore, recombinant KAT2A was able to pull down the recombinant tagged heterodimer XPC/hHR23B in vitro indicating a direct interaction between them (Fig. 4b).

KAT2A is found in two functionally distinct coactivator complexes, SAGA (Spt Ada Gcn5 Acetyltransferase) and ATAC (Ada Two A Containing) $^{20,22}$. These complexes contain either KAT2A or KAT2B. We thus investigated whether the complex containing XPC and KAT2A could be associated with SAGA and/ or ATAC. IP experiments using nuclear extracts from untreated XP-C ${ }^{W T}$ showed that both SAGA and ATAC subunits (TRRAP and ZZZ3, respectively) co-precipitated the complex containing either XPC or KAT2A (Supplementary Figure 4A). IPs of SAGA or ATAC performed with antibodies against the specific subunits TRRAP and ZZZ3, respectively, led to the detection of XPC (Fig. 4c). Interestingly, KAT2B was not immunoprecipitated by XPC although it was detected both in the SAGA and ATAC complexes, indicating the presence of a complex containing XPC and KAT2A HAT in untreated XP-C ${ }^{\mathrm{WT}}$ cells (Fig. 4c). We noticed that the integrity of the ATAC and SAGA complexes was not altered by the absence of XPC in XP-C ${ }^{\text {DEL }}$ cells (Supplementary Figure 4A).

To determine which complex was recruited with XPC at promoters of XPC-dependent genes, we performed ChIP analysis on CCND1 and DAPK1. As expected, neither ZZZ3 (ATAC subunit) nor TRRAP (SAGA subunit) was detected at the promoter of the XPC-negatively regulated CCND1 gene in XP$\mathrm{C}^{\mathrm{DEL}}, \mathrm{XP}-\mathrm{C}^{\mathrm{MUT}}$, or XP-C $\mathrm{CT}$ cells in which KAT2A was not recruited (Fig. 4d, left panel). Interestingly, we only observed enrichment of ZZZ3 and KAT2A at the DAPK1 promoter upon ATRA treatment in XP-C ${ }^{\mathrm{WT}}$ and XP-C $\mathrm{C}^{\mathrm{MUT}}$ cells, while neither TRRAP nor KAT2B was detected (Figs. 4 d, right panel, and $3 \mathrm{~d}$, also Supplementary Figure $4 \mathrm{~B}$ for supplemental genes). The detection of ZZZ3 at the DAPK1 promoter was abolished in XP$\mathrm{C}^{\mathrm{DEL}}$ cells (Fig. 4d, also Supplementary Figure 4B for supplemental genes). In contrast, TRRAP and ZZZ3 were detected at SAGA-targeted RBBP5 and ATAC-targeted SNC16 in XP-C ${ }^{\mathrm{WT}}$ and XP-C ${ }^{\mathrm{DEL}}$ cells, respectively (Supplementary Figure $\left.4 \mathrm{C}\right)^{23}$. 
We also analyzed by ChIP the SAGA vs. ATAC complex recruitment at the $R A R \beta 2$ promoter in shCtrl, shXPC, and shXPA cells. While only ZZZ3 and KAT2A were readily recruited, no TRRAP could be observed at this promoter upon ATRA treatment in shCtrl or shXPA cells. ZZZ3 and KAT2A accumulation was lost in shXPC cells, indicating that the recruitment of the ATAC complex required the presence of XPC (Supplementary Figure 4D).

Altogether, our findings indicated that XPC not only interacts with both ATAC and SAGA but also plays an active role in transcription via the specific recruitment of the ATAC complex to promoters upon transactivation.

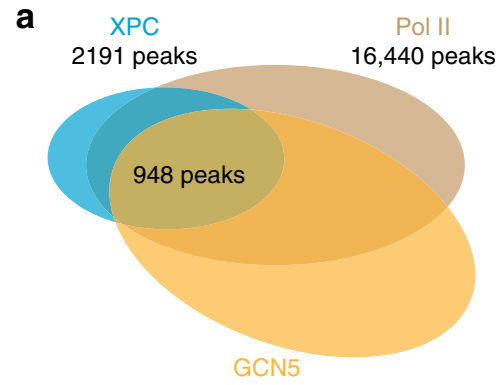

19,758 peaks
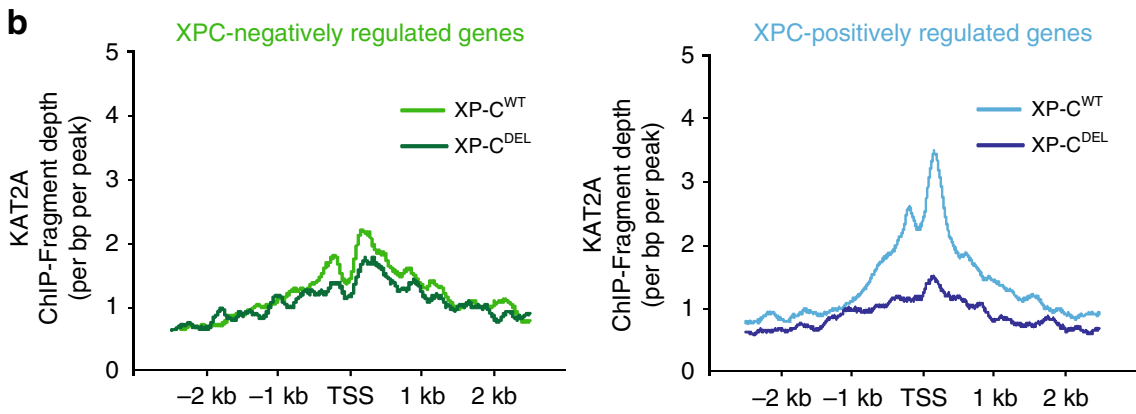

C
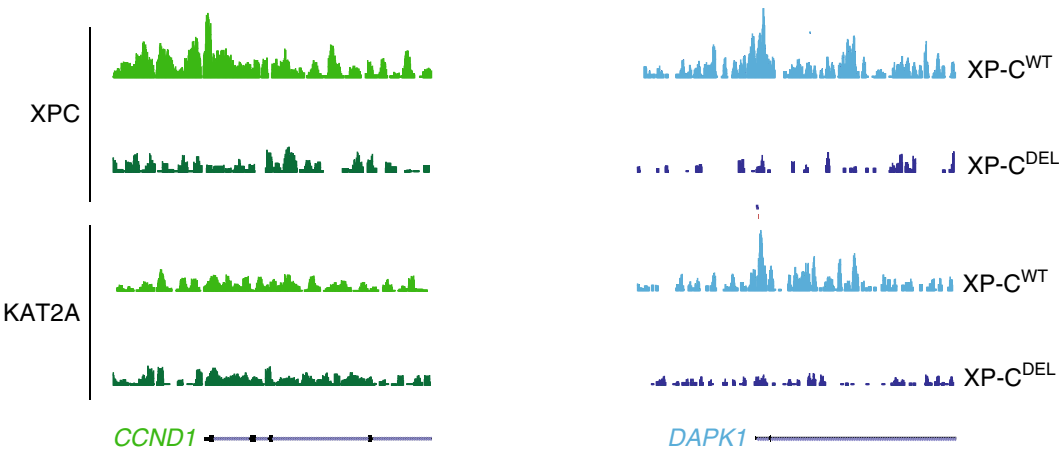

DAPK

d
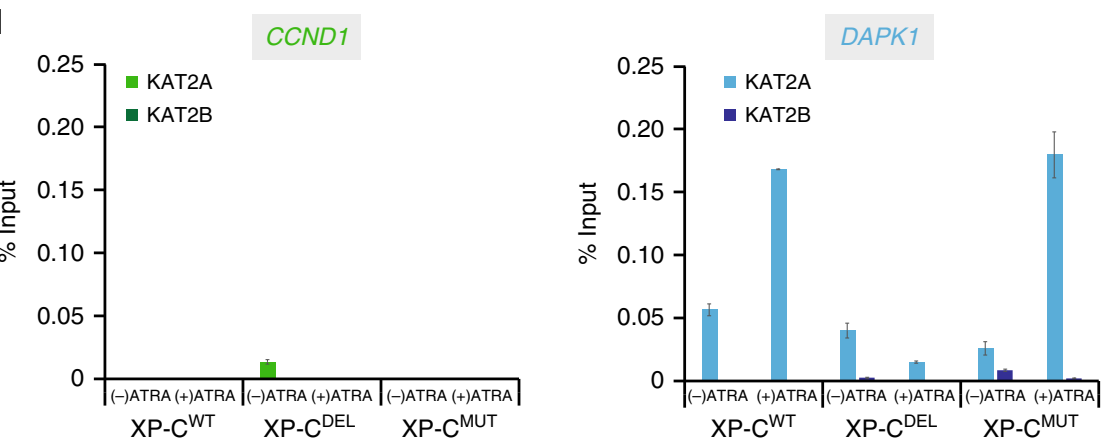

Fig. 3 Association of XPC-bound promoters with KAT2A. a Overlapping of peaks for Pol II and XPC with KAT2A peaks in XP-CWT cells after ATRA induction. These peaks correspond to recurrent peaks found in three independent ChIP-seq experiments. $\mathbf{b}$ Diagrams representing the fragment depth of KAT2A ChIP-seq experiment for XPC-negatively regulated (left panels) and XPC-positively regulated (right panels) genes, as they were previously determined in XP-CWT and XP-CDEL cells. c UCSC genome browser for XPC and KAT2A at CCND1 and DAPK1 promoters as XPC-negatively regulated and XPC-positively regulated genes, respectively. $\mathbf{d}$ Occupancy of KAT2B and KAT2A monitored by ChIP at CCND1 and DAPK1 promoters as XPC-negatively regulated and XPC-positively regulated genes. Error bars represent the standard deviation of three independent experiments 

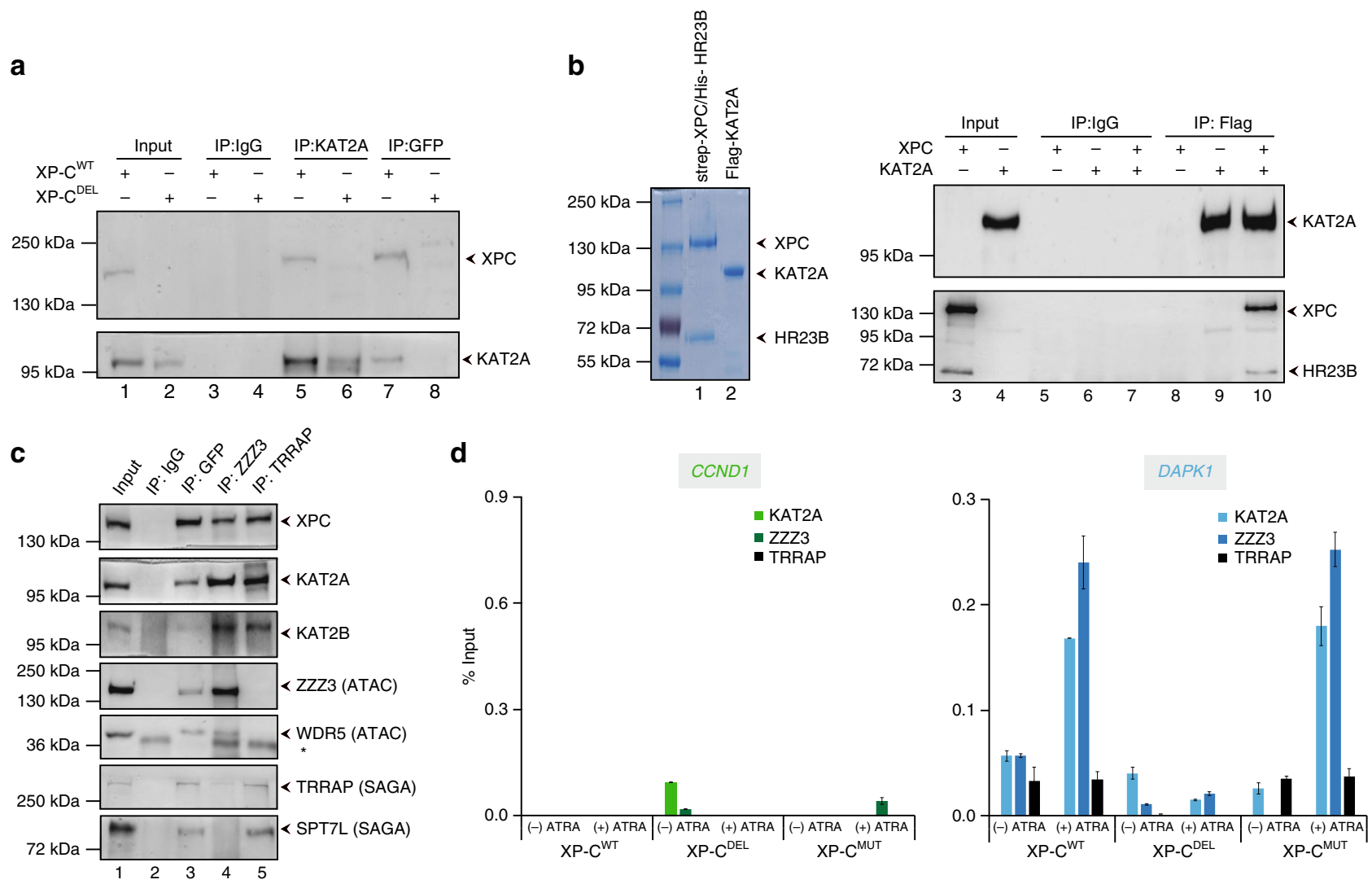

Fig. 4 XPC interacts directly with KAT2A. a Immunoprecipitation performed on nuclear extract of untreated XP-CWT or XP-C $C^{D E L}$ fibroblasts with antibody against GFP, KAT2A, or IgG. b Blue staining of recombinant Flag-KAT2A and duplex strep-XPC/His-HR23B (left panel). In vitro co-immunoprecipitation assay performed by antibodies against Flag tag and IgG with the recombinant protein Flag-KAT2A and the duplex strep-XPC/His-HR23B.

c Immunoprecipitation performed on nuclear extract of untreated XP-CWT fibroblasts with antibody against TRRAP, ZZZ3, GFP, or IgG. Western blot was revealed with antibodies directed against GFP, KAT2A, KAT2B, ZZZ3, WDR5 (ATAC), TRRAP, and SPT7L (SAGA). d ChIP experiment monitoring the recruitment of KAT2B, KAT2A, ZZZ3, and TRRAP at CCND1 and DAPK1 promoters, before and after ATRA treatment. Error bars represent the standard deviation of three independent experiments

XPC is recruited to E2F1-dependent genes after ATRA treatment. In the above results, we established that XPC is present in the ATAC complex via KAT2A, triggering active histone PTMs at the promoter of a subset of genes. We then subsequently searched for specific DNA motifs associated with XPC/ATAC-bound promoters that could explain the recruitment of XPC to these promoters. A GREAT analysis indicated enrichment of E2F1 transcription factor-binding motif at $89 \%$ of the 283 promoters of XPC-positively regulated genes, including DAPK1 and $R A R \beta 2$ (Supplementary Figure 5A). Interestingly, the motif was rarely detected at XPC-negatively regulated genes. We next performed a meta-analysis using E2F1 ChIP-seq data from the ENCODE project by selecting only XPC-positively or -negatively regulated genes, and observed a stronger E2F1 enrichment around promoters for XPC-positively regulated genes (Fig. 5a). ChIP assays on CCND1 and DAPK1 promoters showed the recruitment of E2F1 after ATRA treatment only at the XPC-positively regulated genes in XP-C ${ }^{\mathrm{WT}}$ cells (Fig. $5 \mathrm{~b}$ and also S5B for additional genes). Moreover, the enrichment was lost in the absence of XPC in XP$\mathrm{C}^{\mathrm{DEL}}$ cells while it was still detected in XP-C ${ }^{\mathrm{MUT}}$ cells (Fig. $5 \mathrm{~b}$ and also Supplementary Figure 5B for additional genes).

We next assessed the existence of a complex containing XPC, KAT2A, and E2F1, by carrying out E2F1-immunoprecipitation experiments on nuclear extracts from untreated XP-C $\mathrm{C}^{\mathrm{WT}}$ cells. In these conditions, KAT2A and XPC co-precipitated a complex containing E2F1 (Fig. 5c). We thus investigated whether the detection of the complex, including XPC/hHR23B, KAT2A, and
E2F1 reflected direct interactions between these proteins by using recombinant purified proteins (Fig. 5d). We observed that immunoprecipitation of KAT2A yielded in co-precipitated XPC alone as expected or E2F1 alone as previously showed ${ }^{24}$ (Fig. 5d, lanes 10 and 12) and that a complex containing recombinant and purified XPC/hHR23B, KAT2A, and E2F1 was detected when they were incubated together (Fig. 5d, lane 13).

Our data showed that the presence of responsive element for E2F1 defines the XPC-positively regulated genes and that XPC/ hHR23B, E2F1, and KAT2A can interact to form a tripartite complex.

XPC and E2F1 mediate the recruitment of KAT2A to XPCregulated genes. We next measured the impact of E2F1 and KAT2A on XPC-regulated gene expression and protein recruitment at promoters, by transiently transfecting XP-C ${ }^{\mathrm{WT}}$ cells with siRNA targeting either KAT2A or E2F1 (Fig. 6a). Upon ATRA treatment, the silencing of KAT2A and E2F1 did not disturb the induction of CCND1, while DAPK1 transactivation was abolished (Fig. 6b). ChIP analysis of the KAT2A and E2F1-independent CCND1 promoter showed that their absence did not affect the recruitment of Pol II and XPC or the deposition of H3K9ac upon transcription (Fig. 6c, e, left panel). The silencing of KAT2A did not lead to significant changes in Pol II, XPC, and E2F1 recruitment at the DAPK1 promoter (Fig. 6c, d, right panel). However, the recruitment of Pol II, XPC, and KAT2A decreased upon depletion of E2F1 (Fig. 6c, d, right panel). Both si KAT2A 
a

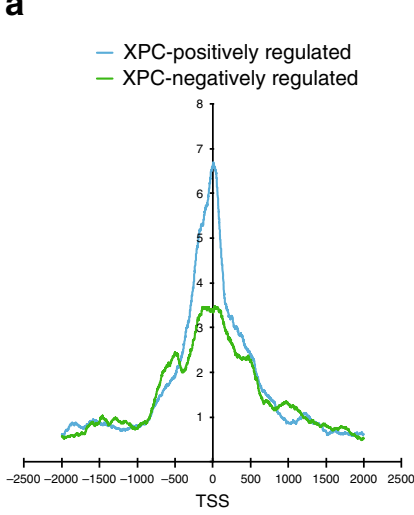

C

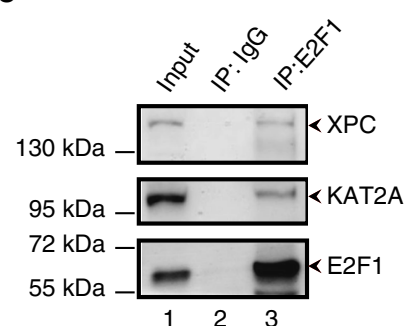

b

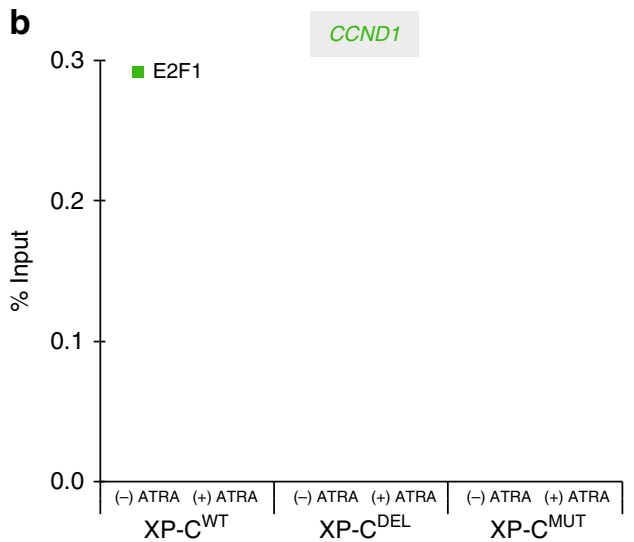

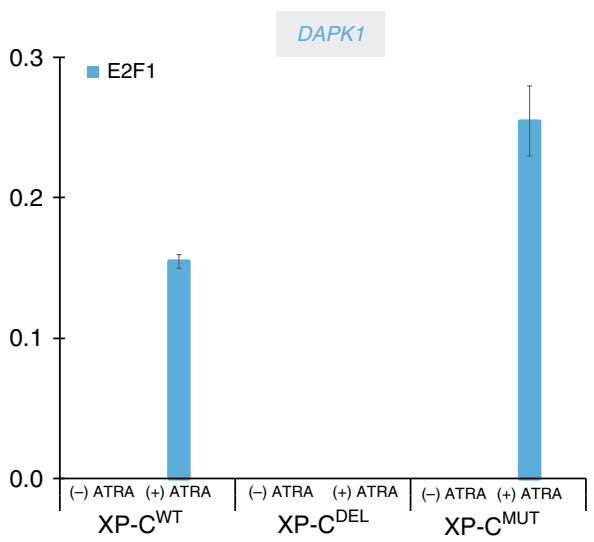

d

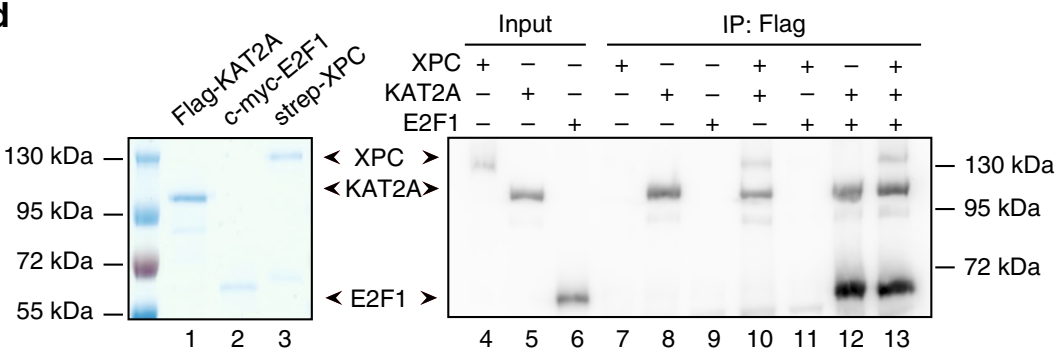

Fig. 5 Ménage-à-trois between XPC, KAT2A, and E2F1. a Diagrams representing the fragment depth of E2F1 from ENCODE ChIP-seq data for XPCnegatively regulated (green) and XPC-positively regulated (blue) genes, as they were previously determined in XP-CWT and XP-C ${ }^{D E L}$. b ChIP experiment investigating the occupancy of E2F1 at CCND1 and DAPK1 promoters, with or without ATRA treatment. Error bars represent the standard deviation of three independent experiments.c Immunoprecipitation performed on nuclear extracts from untreated XP-CWT fibroblasts with antibody against E2F1 or IgG. Western blot was revealed with antibodies directed against E2F1, XPC, and KAT2A. d Left panel; blue staining of recombinant Flag-KAT2A, duplex Strep$\mathrm{XPC} / \mathrm{His}-\mathrm{HR} 23 \mathrm{~B}$, and c-myc-E2F1. Right panel; in vitro co-immunoprecipitation assay performed by antibodies against Flag tag with the recombinant protein Flag-KAT2A, the duplex Strep-XPC/His-HR23B, and c-myc-E2F1

and si E2F1 blocked the acetylation of $\mathrm{H} 3 \mathrm{~K} 9$ at the DAPK1 promoter (Fig. 6e). Similar results were obtained with $R A R \beta 2$ (Supplementary Figure 6).

Together, these results showed that XPC, E2F1, and KAT2A are necessary for the appropriate acetylation of $\mathrm{H} 3 \mathrm{~K} 9$ at the promoter of XPC-positively regulated genes. Moreover, XPC and E2F1 were mutually necessary for their recruitment and drove KAT2A recruitment upon transactivation.

\section{Discussion}

The present work uncovers the genomic distribution and the transcriptional impact of the DNA repair factor XPC. Since XPC is not involved in TC-NER ${ }^{25}$ and our study is performed in the absence of any exogenous genomic stress, the impact of XPC in modulating the transcriptional cell program is independent from its role in DNA repair. In the absence of damage and upon ATRA treatment the lesion sensor XPC was mainly localized around TSSs, collocating with Pol II and regulating positively or negatively around 500 genes. RNA-seq analysis also showed that several hundred genes were deregulated in XPC-depleted cells in the absence of ATRA treatment (Supplementary Figure 7A-B). The comparison between untreated and ATRA-treated conditions in both cells indicated a weak overlap between the different sets of deregulated genes (Supplementary Figure 7C and Supplementary Data 5). The expression of two gene models used in this study was unaffected by the absence of XPC in untreated cells, arguing that XPC only affects their transcription in the complex transcriptional context that represent RAR-dependent transactivation. In accordance to the GO analysis, our results describe phenomenon related to ATRA treatment but the data from untreated cells also showed that our observations are not exclusive to this stimulus. Further experiments will help to characterize the global relevance of the transcriptional role of XPC in transcription and its impact on the pathophysiology of XP-C patients.

For the purpose of clarity and accuracy, we deliberately focused our analysis on ATRA-dependent genes affected by the absence of XPC (representing $46 \%$ of all XPC peaks) although our preliminary data suggested the presence of XPC on other DNA regulatory regions such as enhancers, a phenomenon previously shown for pluripotency regulating genes in ES cells ${ }^{15}$.

The consequences of XPC depletion measured in fibroblasts derived from XP-C patients allowed us to identify, at the genomic scale, its importance for the enrichment of Pol II at TSSs and its specific involvement in the proper distribution of histone PTMs, including $\mathrm{H} 3 \mathrm{~K} 9 \mathrm{ac}$ and $\mathrm{H} 3 \mathrm{~K} 4 \mathrm{me} 3$ marks. Importantly, the analysis of shXPA cells indicated that the enrichment of the histone PTMs upon transcription was specifically related to the presence of XPC.

Using ex vivo and in vitro approaches, we demonstrated that XPC interacted with KAT2A and mediated its recruitment and the related $\mathrm{H} 3 \mathrm{~K} 9 \mathrm{ac}$ deposition at promoter of XPC-positively regulated genes. The co-recruitment of XPC and E2F1 to promoter leads to the recruitment of KAT2A that is present in the SAGA and ATAC coactivator complexes. SAGA and ATAC also contain the close paralog of KAT2A, KAT2B, but only the KAT2A version of these complexes contains XPC. Furthermore, we observed that only ATAC was found to co-localize with XPC upon ATRA treatment so that the interaction of XPC with SAGA and its molecular role remains elusive and requires further investigation. We hypothesize that the SAGA-XPC assembly may target promoters regulated by other specific transcription factors 
and/or by different signaling cascades since ATAC and SAGA are recruited to different stress-induced genes promoters ${ }^{23}$.

Experiments performed in cells depleted in XPA show that $\mathrm{XPC}$ was still recruited to the pre-initiation complex (PIC) while depletion of XPC impaired the recruitment of XPA, indicating that XPC was the first NER factor to be recruited to the PIC. We next sought to identify the signals that triggered the recruitment of XPC to a subset of genes and identified the consensus binding site of E2F1 at $89 \%$ of XPC-activated genes. Using ChIP, we demonstrated that E2F1 and XPC mutually synergized their binding to promoters and consequently contribute both to the recruitment of KAT2A through direct interactions. XPC is then
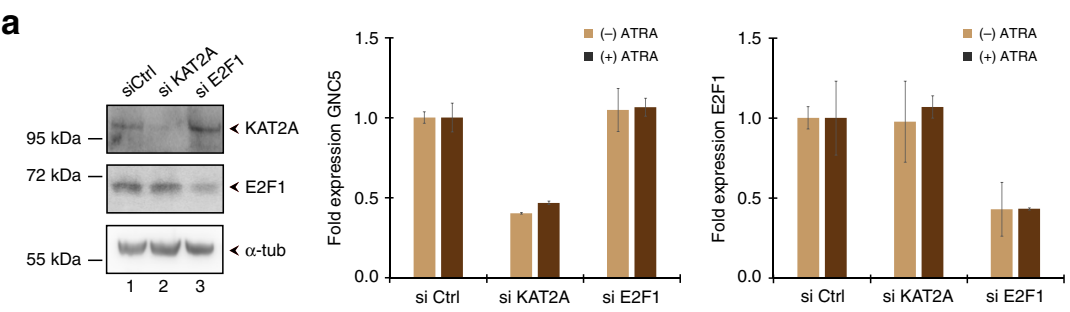

b
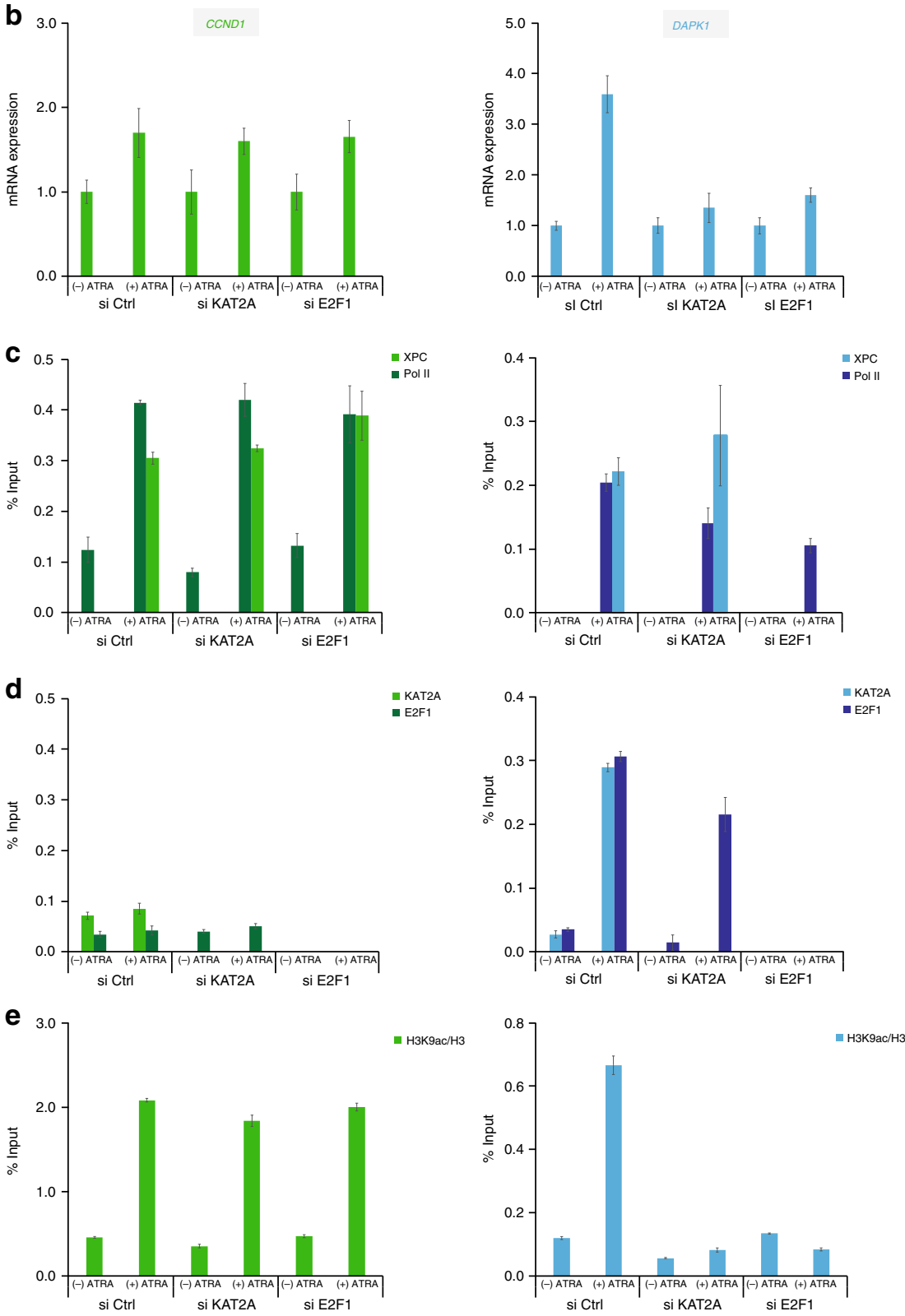

Fig. 6 XPC and E2F1 are mutually necessary for their recruitment and co-operate to recruit KAT2A at promoters upon transcription. a Expression of KAT2A and E2F1 in XP-CWT cells treated with siRNA targeting KAT2A (si KAT2A), E2F1 (si E2F1), or scrambled siRNA (si Ctrl) monitored by western blot (left panel) and qPCR (middle and left panels). b Relative mRNA expression of CCND1 and DAPK1, before and after ATRA treatment in si Ctrl, si KAT2A, and si E2F1 XP-CWT cells. c, d, e Corresponding recruitment of Pol II, XPC (c), KAT2A, and E2F1 (d) as well as histone H3K9ac/H3 (e) at CCND1 and DAPK1 promoters monitored by ChIP. All the error bars represent the standard deviation of three independent experiments 
functioning as a bridge between E2F1 and ATAC, increasing the recruitment of the HAT to XPC-activated genes. As thus, XPC could be considered as a cofactor of mRNA expression in mammalian cells, linking a transcription factor (E2F1) to a coactivator (ATAC). This model also implies that all the mutations affecting XP-C patients are not affecting its transcription function, which is what we observed in XP-C ${ }^{\text {MUT }}$ cells that harbor a punctual mutation (XPC p.Pro334His) affecting XPC NER function without impact on RAR-dependent gene activation. In agreement with this observation, we didn't observe impact of XPC p.Pro334His mutation on XPC/KAT2A interaction.

In addition to E2F1, we cannot exclude the role of additional players for the proper recruitment of XPC to TSS, similarly to TATA-binding protein associated factors (TAFs) that were shown to interact with $\mathrm{XPF}^{26,27}$. Interestingly, among the genes positively regulated by XPC, SET1 encoding one methyl transferase depositing $\mathrm{H} 3 \mathrm{~K} 4 \mathrm{me} 3$ mark has been identified. Concomitantly, a reduced expression of SET1 and a reduced recruitment of the protein at genes activated by XPC were observed in XP-C $\mathrm{C}^{\mathrm{DEL}}$ cells, suggesting an indirect effect of XPC on the H3K4me3 mark (Supplementary Figure 8).

Based on our genome-wide analysis, a large proportion of genes regulated by XPC are related to chromatin and present oncogenic and immunologic signatures. XPC seems to be related to the immune response as demonstrated in two recent studies. First, it has been demonstrated that XPC, through DNA damage, can induce the expression of cytokine like interleukin- 6 possessing pro-tumorigenic effects in lung fibroblasts ${ }^{28}$. Also, multiomic analyses aiming to identify factors and pathways implicated in the cellular response to UV-induced DNA damage have recently shown connections with the immune system ${ }^{29}$. In this study, the analysis of several IFN mRNA expression in XP-CWT and $\mathrm{XP}-\mathrm{C}^{\mathrm{DEL}}$ cells in absence of ATRA treatment indicated higher expressions of IFN beta, gamma, and alpha in cells XPCdepleted that were unrelated to the RAR-dependent pathway (Supplementary Figure 9). Although the dual molecular involvement of NER factors in transcription and DNA repair is now well established, the impact of their deregulation in the pathological context is still elusive, including for the immune response of XP patients. What is more, our data suggest an additional link with transcriptional deficiencies. $D A P K 1$, analyzed in this study, is particularly interesting as it acts as an inhibitor of RIG-I, signaling the necessity to induce the production of type I IFN ${ }^{30}$ and as a tumor suppressor, downregulated in multiple cancer types ${ }^{31}$.

Our comparison of fibroblasts derived from two XP-C patients $\mathrm{XP}_{-} \mathrm{C}^{\mathrm{DEL}}$ and XP-C ${ }^{\mathrm{MUT}}$, bearing XPC p.Arg579* and XPC p. Pro334His mutations, respectively, presenting severe and mild clinical features, respectively, shows deregulation of gene expression only in XP-C $\mathrm{C}^{\mathrm{DEL}}$ cells while GG-NER is impaired in the two cell lines ${ }^{17,18}$. In XP-C ${ }^{D E L}$ cells indeed, the absence of XPC impairs the DNA damage recognition step while in XP$\mathrm{C}^{\mathrm{MUT}}$ cells, the XPC variant can still bind the damaged sites, recruits TFIIH but impedes the stimulation of the TFIIH XPB subunit ATPase activity, leading to a delayed arrival of $\mathrm{XPA}^{17}$. Our observations suggest a correlation between transcriptional default and XP severity although more XP-C cases need to be tested to support this hypothesis. However, the pathological implication of the deregulation of the XPC transcriptional roles remains questioning. Indeed, although XPC has been identified in a transcriptional coactivator complex containing Oct4/Sox 2 that maintains pluripotency of stem cells ${ }^{15}$, it has to be noticed that $\mathrm{XPC}^{-1-}$ mice do not show developmental defects ${ }^{32}$. Also, ablation of the C-terminal region of XPC gene abrogating the interaction sites of XPC with known partners RAD23 and CETN2 has minimal impact on gene expression or pluripotency to contribute to chimeric embryos ${ }^{33}$. The identification of genes and the pathways regulated by XPC will give a more global view of the role of XPC as a Pol II cofactor and will help to identify relevant markers for an early and specific diagnosis, and to anticipate/ predict the cancer risk among the different symptoms. In conclusion, we have uncovered a role for XPC as a regulator of chromatin marks necessary for transcription, redefining the molecular etiology of XP.

\section{Methods}

Cell culture. HeLa Silencix cells (Tebu-Bio), including shCtrl, shXPC, and shXPA cells, MRC-5 fibroblasts (ATCC \# CCL-171), XP-C patient-derived fibroblasts GM14867 (XP-C ${ }^{\text {DEL}}$ ) and GM02096 (XP-C ${ }^{\text {MUT }}$ ), and rescued XP-C (XP-C ${ }^{\text {WT }}$ ) 17,19 (Coriell Institute) were used and cultured in appropriate medium. Twelve hours before ligand treatment, cells were incubated with phenol red-free medium containing charcoal-treated fetal calf serum and $40 \mathrm{mg} / \mathrm{ml}$ gentamycin. Cells were treated with $10 \mu \mathrm{M}$ ATRA (MP).

Reverse transcription and quantitative PCR. Total RNA was isolated from several cell lines using a GenElute Mammalian Total RNA Miniprep kit (Sigma) and reverse transcribed with SuperScript IV reverse transcriptase (Invitrogen). The quantitative PCR (qPCR) was done using the Lightcycler 480 (Roche). The primer sequences for the different genes used in qPCR are indicated in Supplementary Data 3. The mRNA expression of the different analyzed genes represents the ratio between values obtained from treated and untreated cells normalized with the housekeeping GAPDH mRNA.

Chromatin immunoprecipitation. Cells were crosslinked at room temperature for $10 \mathrm{~min}$ with $1 \%$ formaldehyde. Chromatin was prepared and sonicated at $4{ }^{\circ} \mathrm{C}$ for 30 min using sonicator Q800R (Qsonica) as previously described ${ }^{6}$. Samples were immunoprecipitated with antibodies at $4{ }^{\circ} \mathrm{C}$ overnight, and protein G-coated Dynabeads (Invitrogen) were added, incubated for $4 \mathrm{~h}$ at $4{ }^{\circ} \mathrm{C}$, and sequentially washed. Protein-DNA complexes were eluted and decrosslinked. DNA fragments were purified using QIAquick PCR purification kit (QIAGEN) and analyzed by qPCR using a set of primers indicated in Supplementary Data 3.

RNA-seq analysis. Total RNA from XP-C $\mathrm{C}^{\mathrm{WT}}$ and $\mathrm{XP}-\mathrm{C}^{\mathrm{DEL}}$ cells were extracted before or $6 \mathrm{~h}$ after ATRA treatment $(10 \mu \mathrm{M})$ using TRI REAGENT (MRC) and purified by phenol-chloroform extraction. Libraries were prepared with TruSeq Stranded mRNA Sample Preparation kit following guide instruction and subsequently proceed on an Illumina Hiseq 4000 as single-end 50 base reads following Illumina's instructions. Image analysis and base calling were performed using RTA 2.7.3 and bcl2fastq 2.17.1.14. Reads were mapped onto the hg19 assembly of the human genome. Reads count was performed with HOMER v4.8.3(65) and expression was estimated with EdgeR. Genome ontology was performed with The Database for Annotation and Integrated Discovery v6.7 (https://david.ncifcrf.gov).

ChIP-seq analysis. Purified DNA fragments analyzed by ChIP-seq were prepared by using the ChIP-IT High Sensitivity Kit (Active Motif) and the related antibodies. ChIP-seq was performed on an Illumina Hiseq 2500 as single-end 50 base reads following Illumina's instructions. Image analysis and base calling were performed using RTA 1.17.20 and CASAVA 1.8.2. Reads were mapped onto the hg19 assembly of the human genome. Peak detection was performed using MACS (http://liulab.dfci.harvard.edu/MACS/) under settings where the input fraction was used as negative control. Peaks detected were annotated using HOMER (http:// biowhat.ucsd.edu/homer/ngs/annotation.html) as well as TSS protein enrichment comparison. Quantitative comparison of Pol II gene body enrichment was performed using seqMINER (http://bips.u-strasbg.fr/seqminer/). As reference coordinates, we used the MACS-determined peaks or the annotated TSS/TTS of human genes as defined by RefSeq database. Sequence enrichment was performed using RSAT (http://rsat.sb-roscoff.fr) with MACS-determined peaks as reference.

Plasmids and purification of recombinant proteins. PCR products for the entire coding sequence XPC was cloned into pDONOR-207 vector using the Gateway system (Invitrogen) and later cloned in bicistronic plasmid VEAP5317 with hHR23B kindly obtained from A. Poterszman. For recombinant Flag-GCN5 expression in Sf9 cells, the corresponding vector pSK227-KAT2A was kindly provided by $\mathrm{L}$. Tora ${ }^{34}$. PCR product for the entire coding sequence E2F1 was also cloned into pDONOR-207 vector and later sub-cloned in pAC8 vector. Sf9 cells were infected with baculoviruses expressing a FLAG-tagged KAT2A, Strep tactintagged XPC/His-tagged hHR23B, or c-myc-tagged E2F1, and the harvested recombinant proteins were purified as previously described ${ }^{35}$

siRNA transfection. ON-TARGET plus smart pool siRNA control or targeting human KAT2A and E2F1 were purchased from Thermo Scientific and transfected 
in XP-C $\mathrm{C}^{\mathrm{WT}}$ cells at a final concentration of $100 \mathrm{nM}$ using X-tremeGENE siRNA transfection reagent (Sigma-Aldrich) following the manufacturer's protocol.

Co-immunoprecipation. For in vivo co-IPs, nuclear extracts from untreated XP$\mathrm{C}^{\mathrm{WT}}$ and XP-C $\mathrm{CHL}^{\mathrm{DE}}$ cells were prepared as previously described ${ }^{36}$. After GFP-trap or KAT2A, ZZZ3, or TRRAP immunoprecipitation using the appropriate antibodies conjugated to protein G-coated Dynabeads (Invitrogen), followed by extensive washes, was carried out and the different co-precipitated proteins were detected using specific antibodies after immunoblotting.

For in vitro co-IPs, recombinant purified Flag-KAT2A was then incubated with recombinant purified Strep-XPC/His-hHR23B and/or c-myc E2F1 before Flag immunoprecipitation was carried out. After washes, bound proteins were resolved by SDS-polyacrylamide gel electrophoresis and detected by western blot.

Antibodies. Antibodies toward RBP1 (RNA Pol II) (7C2), RAR (9A6), TBP (3G3), XPA (1E11), KAT2A (5GC-2A6), XPG (1B5), ZZZ3 (2616), XPC (2076), and TRRAP (1B3) were produced at the IGBMC. CDK7 (C-19), E2F1 (C-20), and XPC (D-18) antibodies were purchased by Santa-Cruz Biotechnology. H3k4me3 (ab1012), KAT2B (ab12188), and $\alpha$-tubulin (ab15246) antibodies were purchased by Abcam and WDR5 (07-706) antibody from Upstate. Antibodies directed toward KAT2A (A4013) were obtained from Epigentek while those against Spt7L (A302-803A) and hSET1 (A300-803A) from Bethyl. Antibodies against H3K9ac (61251) and H3 (61475) were from Active Motif. Antibodies toward GFP (TP401) and M2-Flag (F1804) were purchased by Torrey Pines Biolabs and Sigma-Aldrich, respectively. For immunoblot, the concerned antibodies were diluted at 1-1000 while 5-10 $\mu \mathrm{g}$ of antibodies were used for ChIP experiments.

Data availability. All relevant data are available from the authors upon request. The accession numbers for the sequencing data reported in this paper are https://www.ncbi.nlm.nih.gov/Traces/study/?acc=SRP148864.

Received: 19 January 2018 Accepted: 25 May 2018

Published online: 04 July 2018

\section{References}

1. Mellon, I., Spivak, G. \& Hanawalt, P. C. Selective removal of transcriptionblocking DNA damage from the transcribed strand of the mammalian DHFR gene. Cell 51, 241-249 (1987).

2. Schaeffer, L. et al. DNA repair helicase: a component of BTF2 (TFIIH) basic transcription factor. Science 260, 58-63 (1993).

3. Feaver, W. J. et al. Dual roles of a multiprotein complex from S. cerevisiae in transcription and DNA repair. Cell 75, 1379-1387 (1993).

4. Barreto, G. et al. Gadd45a promotes epigenetic gene activation by repairmediated DNA demethylation. Nature 445, 671-675 (2007).

5. Schmitz, K. M. et al. TAF12 recruits Gadd45a and the nucleotide excision repair complex to the promoter of rRNA genes leading to active DNA demethylation. Mol. Cell 33, 344-353 (2009).

6. Le May, N. et al. NER factors are recruited to active promoters and facilitate chromatin modification for transcription in the absence of exogenous genotoxic attack. Mol. Cell 38, 54-66 (2010).

7. Cleaver, J. E. Splitting hairs-discovery of a new DNA repair and transcription factor for the human disease trichothiodystrophy. DNA Repair (Amst.) 4, 285-287 (2005).

8. Bootsma, D., Kraemer, K.H., Cleaver, J.E. \& Hoeijmakers, J.H.J. in The Genetic Basis of Human Cancer 2nd edn (eds Vogelstein, B. \& Kinzler, K.W.) 211-237 (McGraw-Hill, New York, 2002).

9. Compe, E. \& Egly, J. M. Nucleotide excision repair and transcriptional regulation: TFIIH and beyond. Annu. Rev. Biochem. 85, 265-290 (2016).

10. Sugasawa, $K$. Regulation of damage recognition in mammalian global genomic nucleotide excision repair. Mutat. Res. 685, 29-37 (2010).

11. Guo, R., Chen, J., Mitchell, D. L. \& Johnson, D. G. GCN5 and E2F1 stimulate nucleotide excision repair by promoting $\mathrm{H} 3 \mathrm{~K} 9$ acetylation at sites of damage. Nucleic Acids Res. 39, 1390-1397 (2011).

12. Takedachi, A., Saijo, M. \& Tanaka, K. DDB2 complex-mediated ubiquitylation around DNA damage is oppositely regulated by XPC and $\mathrm{Ku}$ and contributes to the recruitment of XPA. Mol. Cell. Biol. 30, 2708-2723 (2010).

13. Luijsterburg, M. S. et al. DDB2 promotes chromatin decondensation at UVinduced DNA damage. J. Cell Biol. 197, 267-281 (2012).

14. Le May, N., Fradin, D., Iltis, I., Bougneres, P. \& Egly, J. M. XPG and XPF endonucleases trigger chromatin looping and DNA demethylation fo accurate expression of activated genes. Mol. Cell 47, 622-632 (2012).

15. Fong, Y. W. et al. A DNA repair complex functions as an Oct4/Sox2 coactivator in embryonic stem cells. Cell 147, 120-131 (2011).

16. Cattoglio, C. et al. Functional and mechanistic studies of XPC DNA-repair complex as transcriptional coactivator in embryonic stem cells. Proc. Natl Acad. Sci. USA 112, E2317-E2326 (2015).
17. Bernardes de Jesus, B. M., Bjoras, M., Coin, F. \& Egly, J. M. Dissection of the molecular defects caused by pathogenic mutations in the DNA repair factor XPC. Mol. Cell. Biol. 28, 7225-7235 (2008).

18. Gozukara, E. M. et al. A stop codon in xeroderma pigmentosum group C families in Turkey and Italy: molecular genetic evidence for a common ancestor. J. Invest. Dermatol. 117, 197-204 (2001).

19. Nishi, R. et al. UV-DDB-dependent regulation of nucleotide excision repair kinetics in living cells. DNA Repair (Amst.) 8, 767-776 (2009).

20. Nagy, Z. \& Tora, L. Distinct GCN5/PCAF-containing complexes function as co-activators and are involved in transcription factor and global histone acetylation. Oncogene 26, 5341-5357 (2007).

21. Yang, X. J., Ogryzko, V. V., Nishikawa, J., Howard, B. H. \& Nakatani, Y. A p300/CBP-associated factor that competes with the adenoviral oncoprotein E1A. Nature 382, 319-324 (1996)

22. Wang, L. \& Dent, S. Y. Functions of SAGA in development and disease. Epigenomics 6, 329-339 (2014).

23. Krebs, A. R., Karmodiya, K., Lindahl-Allen, M., Struhl, K. \& Tora, L. SAGA and ATAC histone acetyl transferase complexes regulate distinct sets of genes and ATAC defines a class of p300-independent enhancers. Mol. Cell 44, 410-423 (2011)

24. Lang, S. E., McMahon, S. B., Cole, M. D. \& Hearing, P. E2F transcriptional activation requires TRRAP and GCN5 cofactors. J. Biol. Chem. 276, 32627-32634 (2001)

25. Mu, D. \& Sancar, A. Model for XPC-independent transcription-coupled repair of pyrimidine dimers in humans. J. Biol. Chem. 272, 7570-7573 (1997).

26. Kamileri, I. et al. Defective transcription initiation causes postnatal growth failure in a mouse model of nucleotide excision repair (NER) progeria. Proc. Natl Acad. Sci. USA 109, 2995-3000 (2012).

27. Chatzinikolaou, G. et al. ERCC1-XPF cooperates with CTCF and cohesin to facilitate the developmental silencing of imprinted genes. Nat. Cell Biol. 19, 421-432 (2017)

28. Schreck, I. et al. The nucleotide excision repair protein XPC is essential for bulky DNA adducts to promote interleukin-6 expression via the activation of p38-SAPK. Oncogene 35, 908-918 (2016)

29. Boeing, S. et al. Multiomic analysis of the UV-induced DNA damage response. Cell Rep. 15, 1597-1610 (2016).

30. Willemsen, J. et al. Phosphorylation-dependent feedback inhibition of RIG-I by DAPK1 identified by kinome-wide siRNA screening. Mol. Cell 65, 403-415. e8 (2017).

31. Kissil, J. L. et al. DAP-kinase loss of expression in various carcinoma and B-cell lymphoma cell lines: possible implications for role as tumor suppressor gene. Oncogene 15, 403-407 (1997).

32. Sands, A. T., Abuin, A., Sanchez, A., Conti, C. J. \& Bradley, A. High susceptibility to ultraviolet-induced carcinogenesis in mice lacking XPC. Nature 377, 162-165 (1995)

33. Ito, S., Yamane, M., Ohtsuka, S. \& Niwa, H. The C-terminal region of Xpc is dispensable for the transcriptional activity of Oct3/4 in mouse embryonic stem cells. FEBS Lett. 588, 1128-1135 (2014).

34. Demeny, M. A. et al. Identification of a small TAF complex and its role in the assembly of TAF-containing complexes. PLoS ONE 2, e316 (2007).

35. Singh, A., Compe, E., Le May, N. \& Egly, J. M. TFIIH subunit alterations causing xeroderma pigmentosum and trichothiodystrophy specifically disturb several steps during transcription. Am. J. Hum. Genet. 96, 194-207 (2015).

36. Nagy, Z. et al. The metazoan ATAC and SAGA coactivator HAT complexes regulate different sets of inducible target genes. Cell. Mol. Life Sci. 67, 611-628 (2010).

\section{Acknowledgements}

We are grateful to A. Poterszman and L. Tora (IGBMC) for the VEAP5317 and KAT2A constructs, respectively, to members of our team, and to Didier Devys (IGBMC) for fruitful discussion. Sequencing was performed by the IGBMC Microarray and Sequencing platform, a member of the "France Génomique" consortium (ANR-10-INBS-0009) This study was supported by l'Association de la Recherche contre le Cancer (ARC no. SL220130607082) and the National Research Foundation of Korea for International Collaboration. This study was supported by the Ligue contre Le cancer (CCIR-GE 2015). This study was supported by the grant ANR-10-LABX-0030-INRT, a French State fund managed by the Agence Nationale de la Recherche under the frame program Investissements d'Avenir ANR-10- IDEX-0002-02. B.B was supported by the fondation pour la recherche médicale (FDT20160736467). M.S. was supported by le prix d'encouragement à la recherche de la province Sud (Nouvelle Calédonie).

\section{Author contributions}

B.B., I.I., M.S., Z.N., A.L. A.C., M.B., and N.L.M. conducted experiments and analyzed data. N.L.M., F.C., and J.-M.E., conceived the project, supervised the research, and wrote the manuscript with input and editing from all the authors. 


\section{Additional information}

Supplementary Information accompanies this paper at https://doi.org/10.1038/s41467018-05010-0.

Competing interests: The authors declare no competing interests.

Reprints and permission information is available online at http://npg.nature.com/ reprintsandpermissions/

Publisher's note: Springer Nature remains neutral with regard to jurisdictional claims in published maps and institutional affiliations. (c) (i) Open Access This article is licensed under a Creative Commons Attribution 4.0 International License, which permits use, sharing, adaptation, distribution and reproduction in any medium or format, as long as you give appropriate credit to the original author(s) and the source, provide a link to the Creative Commons license, and indicate if changes were made. The images or other third party material in this article are included in the article's Creative Commons license, unless indicated otherwise in a credit line to the material. If material is not included in the article's Creative Commons license and your intended use is not permitted by statutory regulation or exceeds the permitted use, you will need to obtain permission directly from the copyright holder. To view a copy of this license, visit http://creativecommons.org/ licenses/by/4.0/.

(C) The Author(s) 2018 